\title{
Process improvement through Root Cause Analysis
}

\author{
G. Damele*, G. Bazzanas, F. Andreis*, F. Aquilio*, S. Arnoldi*, \\ E. Pessi ${ }^{\circ}$ \\ * Italtel SIT, 20019 Settimo Milanese (Italy) \\ Tel: +3924388 1; Fax: +39243888705 \\ $\$$ at the time of writing: Etnoteam SpA \\ at the time of final submission: Onion Srl, 25020 Brescia (Italy)
}

Tel: +3930 3581510; Fax: +3930 3581525; email: gb@onion.it

- Etnoteam SpA, via A. Bono Cairoli 34, 20127 Milano (Italy)

Tel: +392261621 Fax: +392261 107 55; email: epessi@etnoteam.it

\begin{abstract}
Italtel SIT BUCT Linea UT has been conducting a software process improvement program since 1991, following some of the most widespread paradigms, including: process certification in accordance with ISO 9001, Process Quality Management and Improvement (PQMI), process assessment in accordance with Bootstrap. After four years, the improvement program has brought to tangible quantitative benefits and to the reaching of a stable maturity level 3 . Among the lines of actions that have been set up to feed and care the continuous improvement program, a particular emphasis has been put on the experimentation of defect prevention techniques. In this context, a Root Cause Analysis (RCA) program has been implemented, focusing both on failures and on scheduling slippages.

The paper is focused on the experiences matured, dealing with the following topics:

- basics of the Italtel SIT BUCT Linea UT process improvement program;

- summary of the benefits achieved and of the challenges set for the next future;

- the adoption of Root Cause Analysis techniques for the sake of defect prevention;

- the results from the application of RCA to the failures found in operation;

- the extension of RCA to the investigation of scheduling slippages;

- the feed-back on the development process.

The goals, the activities, the results and the findings are presented in adherence with a PlanDo-Check-Act (PDCA) paradigm and are supported by quantitative data. The conclusions of the study can be summarised as follows:

- From a technical point of view, RCA has contributed to a better understanding of the development/ testing practices and to the adoption of a number of corrective actions.

- From a methodological point of view, RCA has proven to be powerful in singling out improvement opportunities; for this reason the software producing unit is aiming at its extension and standardisation.
\end{abstract}

\section{Keywords}

Process improvement, RCA, PDCA, Bootstrap. 


\section{THE PROCESS IMPROVEMENT PROGRAM UNDERGOING AT ITALTEL SIT BUCT LINEA UT}

Italtel designs, manufactures, markets and installs systems and equipment for private and public networks. This software producing unit is committed, among other products, to the development and maintenance of the Linea UT telecommunication switching system (Italtel, 1995). At the end of 1994, over 15 million Linea UT lines, 1000 exchanges and 2000 RSU (Remote Subscriber Units) were operating in 18 countries world-wide.

As part of a company wide quality program (Maggi, 1995), Italtel SIT BUCT is strongly committed to the excellence of quality in delivered products and internal processes, in adherence to Process Quality Management and Improvement (PQMI) principles (AT\&T, 1989). In this context the main goal of the improvement program was to adopt a Plan-Do-Check-Act (PDCA) scheme able to check and measure the products and the development process in a quantitative way in order to single out, implement and monitor the improvement opportunities. The improvement program has its roots in the Quality Management System (Italtel, 1993) and is supported by quality planning activities and by a measurement system that has been applied since 1991 (Damele, 1993).

Italtel SIT BUCT holds ISO 9001 certification (ISO, 1990) since April 1992; since 1993 the certificate has been recognised at European level by ITQS (Souter, 1992). Now the company is moving towards Total Quality Management assessment schema recognised world-wide (ASQC, 1995).

In order to drive and monitor process improvement also in software, process assessments (performed with the Bootstrap methodology) take place at regular time intervals. Bootstrap (IEEE, 1993) (Kuvaja, 1994) is an European assessment method, based on the Software Engincering Institute Capability Maturity Model - SEI CMM (Paulk, 1993) reference scheme and questionnaire, but extended with concepts widely adopted by European companies, namely: ISO 9000 quality standards and the European Space Agency's process models. The resulting assessment approach yields capability profiles at a fine granularity level and can be applied to both organisations and projects. Maturity levels are divided into quartiles, and maturity determination can be applied to a wide set of capability factors divided into three groups: organisation, methodology, technology. For the most part, the SEI's maturity levels are still recognisable and the assessments are largely compatible with CMM ones.

The latest process assessment (Damele, 1995), undertaken in 1994, concluded that Italtel SIT BUCT Linea UT has progressed to a maturity level 3.5 at Software Producing Unit (SPU) level and 3 at project level, with many activities reaching level 4 grading. Improvements include almost all areas with respect to the baseline of the first assessment, indicating that the SPU has reached a good positioning, as detailed in Fig. 1, that shows the maturity levels of the various practices both from a global (or in other terms: SPU or QMS) perspective and a project perspective, with details also of the maximum maturity level of each area, in order to highlight the difference between the current status and the optimising level (the highest grading). 


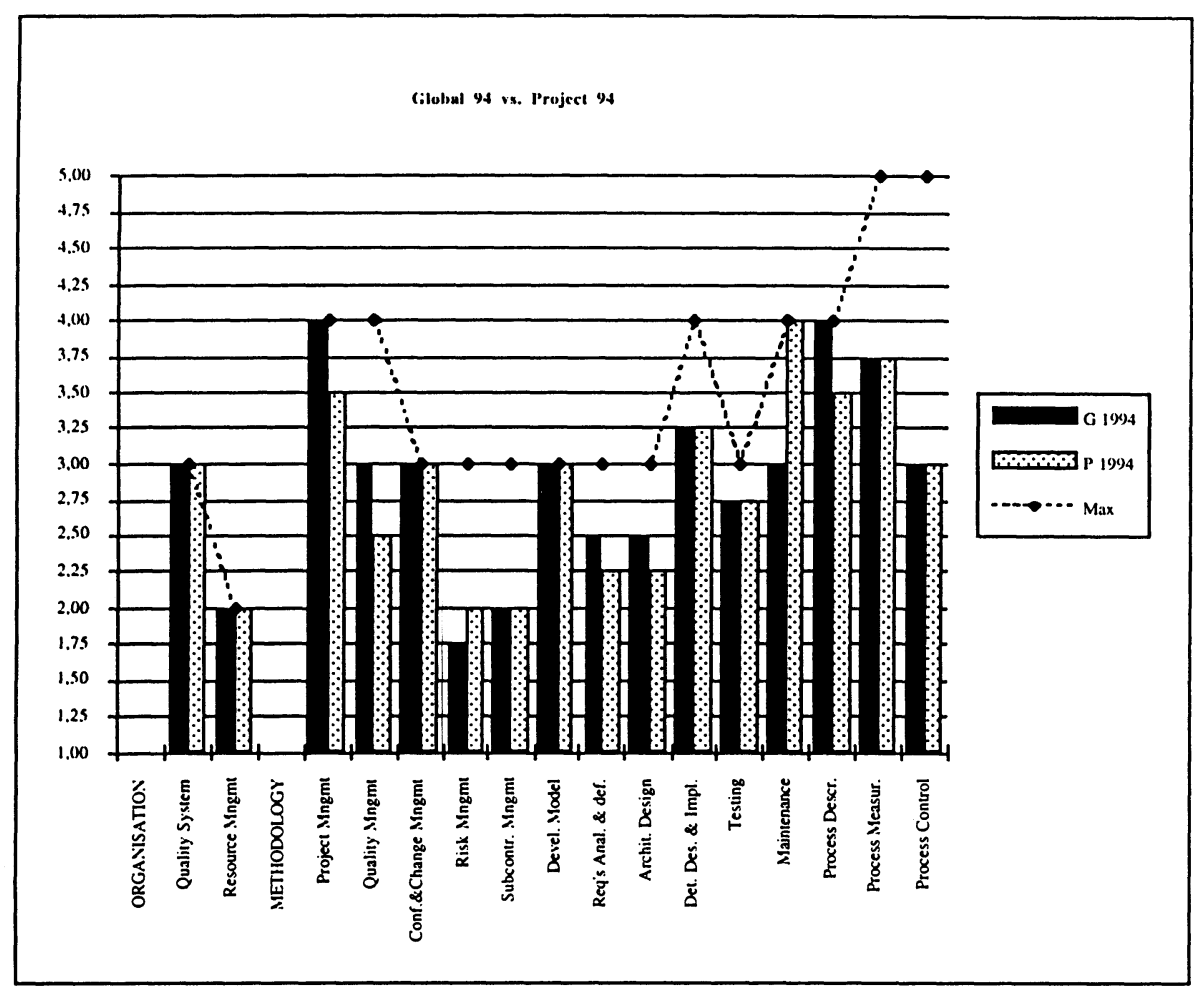

Figure 1 - Italtel SIT BUCT Linea UT Assessment results in 1994 according to Bootstrap.

\section{RESULTS ACHIEVED FROM THE IMPROVEMENT PROGRAM}

The process improvement program has given valuable contributions in reaching the goals of the software producing unit; results have been tracked and analysed in a quantitative way, thanks to the early adoption of a measurement system. The metrics have been defined in accordance with the goals of the SPU: timeliness, reliability, documentation, resources. Indicators are collected and analysed from two different points of view: the customer and the project. Table 1 summarises the indicators defined at customer and project levels and their relationships with the goals of the SW producing unit (for a detailed definition of indicators and basic data, the interested reader is referred to (Damele, 1993)).

Table 1 - Relationships between indicators and goals of the metrics program

\begin{tabular}{lll}
\hline Goal & Indicators relevant for the customer & Indicators relevant for the project \\
\hline Timeliness & - Timeliness for the customer & - Timeliness for the project \\
& & - Efficacy \\
\hline Reliability & - Fault Rate in operation & - Fault Rate in testing \\
& - Testing Effectiveness & - Fault Density \\
\hline Documentation & - Documentation for the customer & - Documentation for the project \\
\hline Resources & & - Productivity \\
\hline
\end{tabular}


As detailed in (Damele, 1995), the following results can be reported after four years of process improvement:

- Timeliness for the customer has always been achicved; moreover, if we consider the contribution of process improvement it can be shown that timeliness capabilities have been improved by $10 \%$.

- Reliability in operation has reached a fault density of less than 1 fault per $10 \mathrm{KLOC}$, with an improvement of $54 \%$; the net contribution of process improvement is tracked by the 'testing effectiveness' indicator, that has increased by $7 \%$.

- Documentation for the product is quickly increasing and has reached its goals, without negatively affecting other indicators (in particular: timeliness issues).

- Productivity goals have been largely reached and we can observe better performance by a factor of around $65 \%$ even if, considering the difference between expected values and actual ones, we come to the conclusion that this is the area where benefits from process improvement are less tangible.

\section{ROLE OF RCA WITHIN THE IMPROVEMENT INITIATIVE}

Fig. 2 shows the path that has been done so far in terms of activities undertaken and maturity levels reached since 1990 .

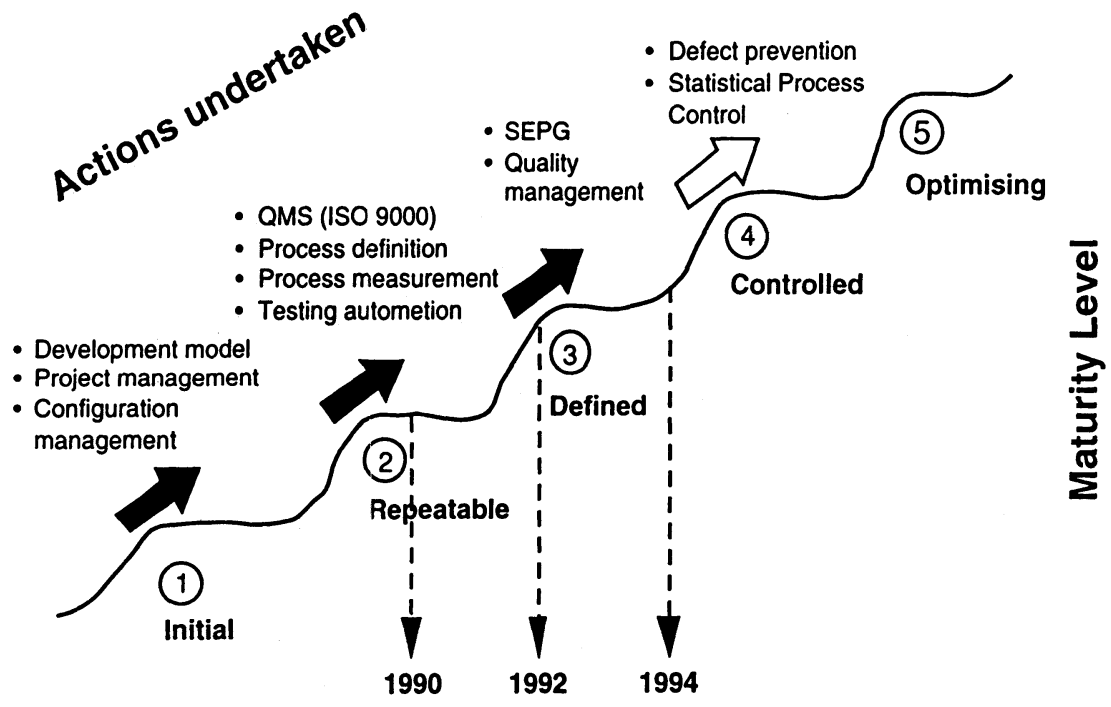

Years

Figure 2 - Italtel SIT BUCT Linea UT path towards process improvement.

Defect prevention is, together with Statistical Process Control, the current challenge for growing the capabilities of the producing unit. As a consequence, Root Cause Analysis has been investigated for adoption. 


\section{BASICS OF ROOT CAUSE ANALYSIS}

\subsection{Goals of RCA}

As well described in (Briand, 1995), casual analysis (or, in other terms, Root Cause Anlaysis) is a technique appropriate to identify the causes and inner mechanism that lead to costly or risky problems related to the quality of the delivered products or the efficiency of the development process. As a consequence, Root Cause Analysis (RCA) has been chosen as the technique supporting cause-cffect modelling, in conjunction with the well-known classifications proposed by Ishikawa (Ishikawa, 1991). The goal of RCA is the analysis of the problem occurred for evaluating why it happened, in order to identify the root causes of the problem, not simply the symptoms. In so doing we can derive a diagnosis for fixing the defects in the process or materials that caused the problem in the first place. RCA techniques are based on the observation that SW problems (faults, scheduling slippages, budget overheads) are not only symptoms of a problem in the product, but also in the process that created it; as a consequence, the root cause might involve the processes, inputs, environment or people involved. In order to get the knowledge and remove the root cause (thus preventing future problems of a similar nature) what is needed is an extra effort to go back and determine why the problem was created in the first place.

The aim of RCA is the improvement of the process through the analysis of problems, by means of a set of activities including:

- Investigation of where and why problems have been introduced.

- Investigation of where the problems have been detected and why they were not detected earlier.

- Formulation of recommendations to eliminate or reduce the incidence of similar errors in subsequent development projects.

The preliminary condition for the application of RCA is the traceability of the processes. In fact, for an objective approach, the processes shall be defined and described in order to perform analysis on the project documentation.

A second condition for the application of RCA is that it must be supported by an ad-hoc group, that has the duty of classifying the possible causes and to support developers in performing the analysis; as a matter of fact you must not stop at the first cause but you have to go back to the root cause, by means of repetitive "Why?" (as taught by Japanese approaches to RCA, known as "Naze-Naze", that simply means "Why? Why?").

\subsection{RCA in the context of PDCA}

Whenever a process improvement initiative is decided, it should be driven in accordance with a PDCA strategy (Ishikawa, 1991).

The PDCA problem-solving approach is very simple in its foundations:

- Plan: identify and analyse the problem;

- Do: deploy countermeasures;

- Check: verify root causes decreased and targets met or exceeded;

- Act: sustain improvements and make them part of the QMS.

The application of the overall PDCA scheme for the RCA activities is quite time-consuming and will be tracked by the measurement system applied to the projects that will benefit of the countermeasures decided after the analysis of RCA findings.

But a PDCA scheme can be applied also to the RCA activity itself; in particular, adapting the general procedures to perform casual analysis (Collofello,1993) (Nakajo, 1991), the various steps have to be intended in the following way:

- Plan:

- analysis of quality indicators in order to single out improvement opportunities;

- $\quad$ selection of the data sample onto which RCA should be applied;

- definition of the set of possible causes to investigate; 
- Do:

- data collection;

-. validation of collected data;

- Check:

-• analysis of data with usage of Ishikawa diagrams;

- interpretation;

-* cost-effectiveness analysis of possible actions aiming at eliminating root causes;

- Act:

-• cvolution of the QMS in order to incorporate process improvement actions agreed.

In the following such PDCA scheme will be used for describing the application of RCA to failures, that is to say:

- planning RCA of failures;

- doing RCA of failures;

- checking the results from RCA;

- acting in adherence with RCA recommendations.

\section{ROOT CAUSE ANALYSIS OF FAILURES}

\subsection{Planning RCA of failures}

\subsubsection{Analysis of quality indicators in order to single out improvement opportunities}

Table 2 details the values in 1992 and 1994 for the indicators of the measurement system referring to reliability issues. For each indicator, the metrication unit is stated, together with the goal that was established at the beginning of the improvement program.

Table 2 - Values for the customer-relevant indicators affecting reliability

\begin{tabular}{lllll}
\hline Indicator & Unit & Goals & 1992 & 1994 \\
\hline Fault density in operation Faults/Kloc & $<0.12$ & 0.149 & 0.08 \\
\hline Fault rate in operation & Faults/Week & $<10$ & 4.02 & 6.22 \\
\hline Testing effectiveness & \% of faults pre-release & $>80$ & 77 & 84.1 \\
\hline
\end{tabular}

The indicators can be interpreted in the following way:

- the challenging goals of the improvement program have been attained;

- fault density in operation has significantly improved;

- testing effectiveness has also improved;

- failure rate has worsened (keeping in any case its goal).

The last point is worth discussing: despite the fact that the process has significantly improved, the customer perceives in field more failures per week; this is due to the fact that the size of released products grows so much that improvement in fault density is overridden. This means that process improvement has to continue and even to accelerate in order to compensate the unavoidable growth of applications released!

Hence the need to understand, by means of RCA, where to focus the additional improvement efforts, that should concentrate on bettering fault density up to the point that the fault rate becomes at least stable. 


\subsubsection{Selection of the data sample onto which RCA should be applied}

The RCA was applied to a set of failures coming from the field and selected on the basis of their severity.

The reasons for this choice are:

- The field failures are the most relevant aspect of the quality perceived by the customer.

- The field failures are the most important to analyse because they have not been captured neither by internal tests nor by validation tests with the customer.

- In accordance with an analysis made in the specific environment, the cost to found, fix and validate failures from field is 70 times more than the cost to found, fix and validate failures found during system test (Tosi, 1994).

On the bases of severity and of fair distribution across development areas, 98 failures out of 313 were selected for analysis. The sample is thus considered to be fairly representative and of statistical validity.

\subsubsection{Definition of the set of possible causes to investigate}

In order to keep full control of the experiment, the analysis focused only on the internal processes, while processes mastered by external entities (such as: technical assistance, system requirement specification, acceptance) were not analysed.

A team of experts in the different activities of the development process was set-up with the aim to prepare a questionnaire including the possible causes of errors; in particular, a distinction was made between the causes for the injection of the error and the causes for not detecting the problem.

A questionnaire was prepared for each phase of the development life-cycle for a total of several hundreds of questions. It has to be underlined that, in order not to influence the subsequent collection of results, questions were not clustered in accordance with root causes but presented in a flat format.

\subsection{Doing RCA on failures}

\subsubsection{Data collection}

The data was collected by means of direct interviews with designers involved in development, testing and fixing of the faulty features. Interviews fully adopted a back-tracking strategy: each time a selected cause had roots in a previous phase of the development life-cycle, the analysis jumped on that activity, starting a new interview with the appropriate questionnaire together with the people in charge of the activity under analysis. For this reason, starting from 98 failure, more than 300 interviews were done, involving 200 persons at varying responsibility levels. In order to select the right people to interview, the planning documents were taken into account.

\subsubsection{Validation of collected data}

Before analysing the data collected, a validation step was performed. The goal of validation was not to review the results in order to pre-process them but rather to allow the possibility of uncovering additional causes. For this reason the development area managers were asked to give their view of the causes onto which designers had already expressed their point of view. It was not seldom the case in which opinions differed, sometimes also utterly. In these cases, the analysis of inconsistencies between the different points of view proved invaluable in suggesting additional causes. 


\subsection{Checking the results from RCA}

\subsubsection{Analysis of data with usage of Ishikawa diagrams}

The results of the interviews were stored into a database; the data was then analysed with usage of SAS, a statistical analysis tool. In order to identify the root causes of the introduction and the missing detection of failures, "cause-effect" Ishikawa diagrams were adopted (Ishikawa, 1991). Such diagrams are very useful for presenting RCA results since they show the relationships between the effects (that is to say, the symptoms observed) and the causes. They are also called fishbone diagram owing to the resemblance to a fish's skeleton, with the head being the effect and the bones the cause at various levels of nesting.

\subsubsection{Root causes for error injection}

The first analysis step consisted in the identification of the process where the error was injected in the first place. Figure 3 shows the percentage distribution across the various processes identified.

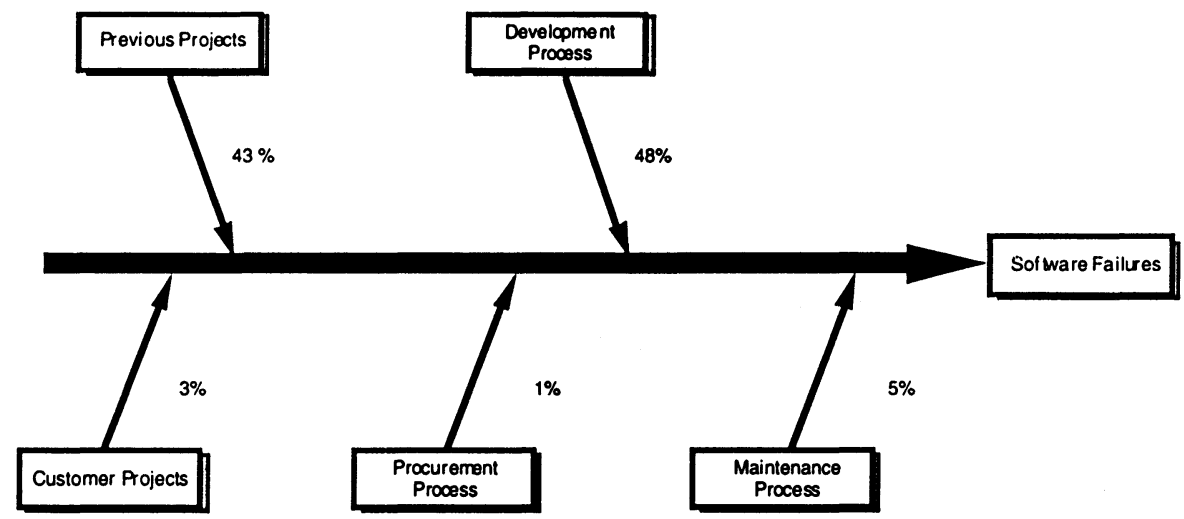

Figure 3 - Processes responsible for fault injection.

This step brought to an interesting result:

- The biggest contribution comes (as expected) from development activities.

- About $43 \%$ of problems were due to failures injected in the previous project and inherited in the current one; this was interpreted as due to the fact that the previous release experienced a modest usage on field and thus many problems were not observed and detected.

- Very few problems were due to change management.

- The impact of external processes (procurement and definition of requirements) is negligible.

Zooming into the development activities, the biggest contribution was found from the coding activities (83\%), far exceeding the impact due to errors injected during functional specifications $(17 \%)$. Within coding activities (known also as 'block design') the causes were grouped into four categories: People, Methods, Documentation, Development Environment \& Tools. From the analysis of the percentage of errors assigned to each category (see Figure 4) for a simplified representation the most critical category was identified as "Method", with lack of time as the most recurrent cause (as already reported by similar experiences, see (Furuyama, 1993)). "People" issues were the second cause of errors, with aspects like "human factors", "no adoption of coding rules" and "wrong interpretation of specs" as the prevailing typologies. 


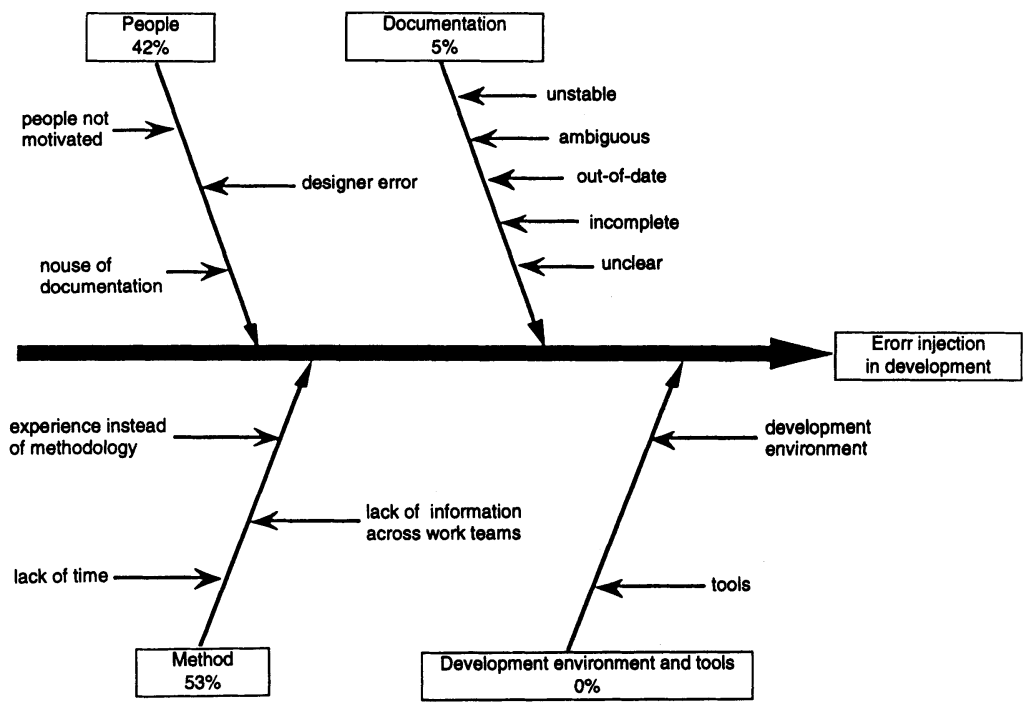

Figure 4 - Categorisation of causes for error injection during coding.

\subsubsection{Root causes for missed error detection}

Concerning the reasons for not being able to discover the errors, $57 \%$ of faults should have been discovered during system test (also known as function test) and $33 \%$ in integration (component) test, with the remaining $10 \%$ that ought to have been discovered during change management activities. The following picture shows the categorisation of causes as far as system test is concerned.

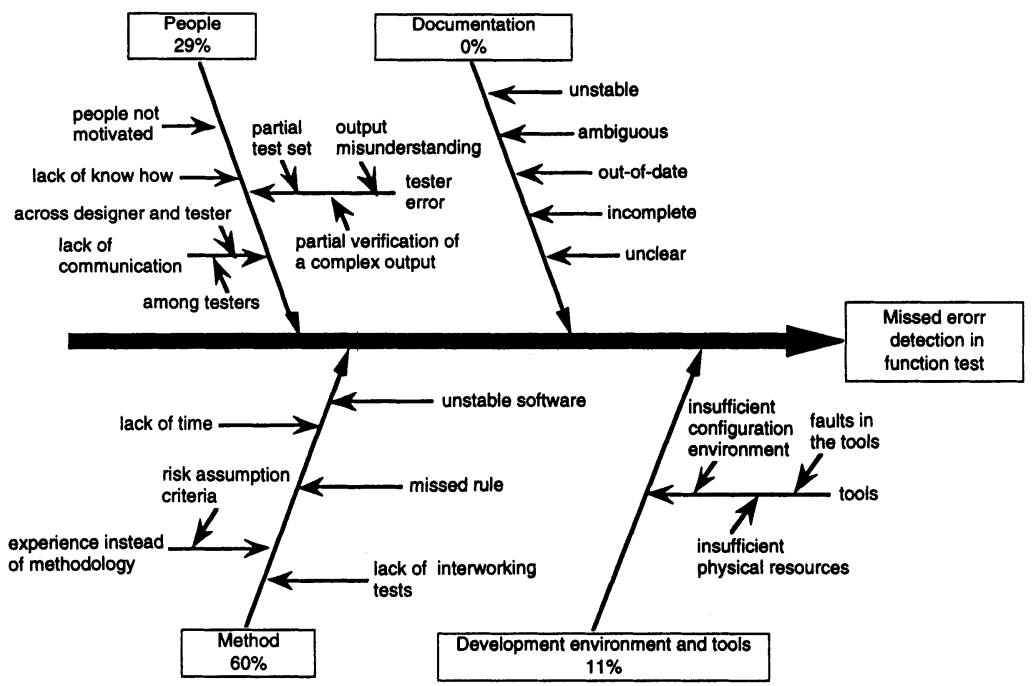

Figure 5 - Categorisation of causes for missed error detection during system test. 


\subsubsection{Interpretation of results}

The following Pareto chart summarises in the overall the root causes of failures clustered across the four identified categories.

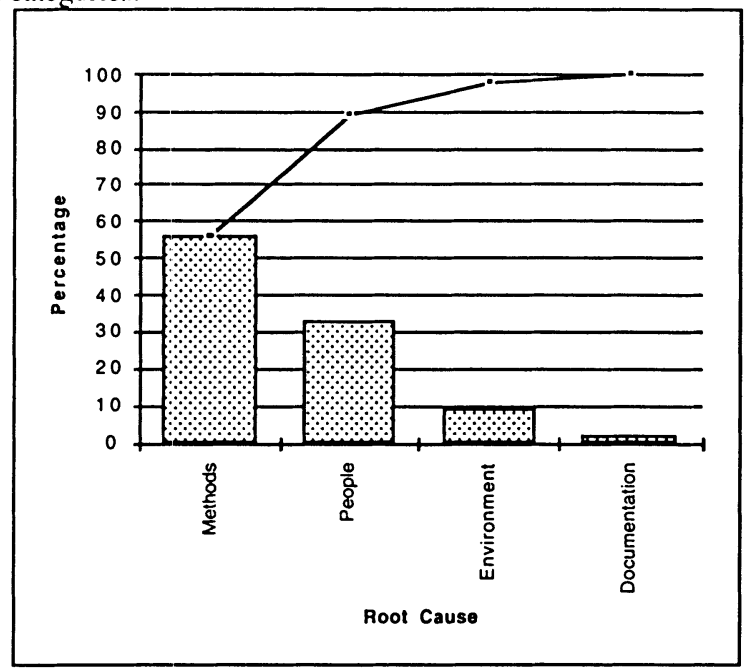

Figure 6 - RCA of failures: overall aggregation of results.

Several interpretations were very useful, among which:

- the high impact of problems injected in previous projects;

- the major role of coding in error injection;

- the major role of system test in missed error detection;

- the clusters of "method" and "people" causes;

- the high impact of the following causes, in decreasing order of priority: "lack of time", "lack of adoption of coding rules", "test case selection strategy", "usage of experience instead of rules", "missing reviews".

Such causes were elaborated in order to take preventive and corrective actions, as described in the "Act" paragraph.

\subsubsection{Cost-effectiveness analysis}

The following table summarises the effort spent in performing RCA of failures, including data collection, data analysis, meetings, interviews, reporting and all other kinds of activities.

Table 3 - Effort spent in RCA of failures

\begin{tabular}{ll}
\hline Activity & Effort in person-days \\
\hline Plan - analysis of quality indicators & 5 \\
\hline Plan - selection of the data sample & 10 \\
\hline Plan - definition of possible causes & 40 \\
\hline Do - data collection & 400 \\
\hline Do - validation & 80 \\
\hline Check - data analysis & 100 \\
\hline Check - interpretation & 50 \\
\hline Check - cost-effectiveness analysis & 15 \\
\hline Act - evolution of QMS & not available yet \\
\hline TOTAL & 700 \\
\hline
\end{tabular}


Such figures correspond to about $1,5 \%$ of the overall effort spent for the project to which RCA was applied. Part of the effort is due to the lack of previous specific experiences in RCA (especially in the definition of possible causes), but the biggest part (around 70\%) was spent in data collection and validation. The effort needed for such activities might be largely reduced in case RCA is performed as soon as the failure is detected, using SW factory tools for declaring the root cause.

Even if precise effort estimates for the 'Act' phase are not yet available, first analyses show that a positive Return On Investment will be reached in case the resulting preventive actions will bring to a $2 \%$ improvement in the indicator 'Testing Effectiveness' and to a stabilisation of the indicator 'Failure Rate'. In any case, RCA is likely to be introduced steadily in the working practices only if it will be possible to run it as part of the daily routine work, with an effort overhead limited to $0,5 \%$ of the overall project budget.

\subsection{Acting in adherence with $\mathrm{RCA}$ recommendations}

The ultimate goal of RCA is process improvement. For this task an expert working group was set-up to evaluate and propose a set of process improvement actions. Improvement actions have been decided at three levels:

- improvement to the development/ testing process;

- improvement to specific technical aspects;

- improvement to the mechanics of RCA.

As far the development/ testing process are concerned, the following improvement actions were decided:

- More careful co-ordination between integration testing and system testing activities, in order to ensure the maximum synergy and the minimum overlap.

- Widening of test automation, in order to overcome scheduling problems during test execution and to force the application of standard rules instead of personal experiences.

- Controlled adoption of the risk management criteria used in deciding the accuracy of integration tests and in selecting system test cases, by means of the adoption of process waivers that have to be authorised by appropriate management levels (for a detailed presentation of this method and of the related "Process Standardisation" indicator, the interested reader is referred to (Damele, 1995)).

Concerning specific technical aspects, the following actions have been agreed:

- extension of training on coding practices and testing guidelines;

- review of system test documents in order to include non-regression tests aiming at covering the most frequently emerged issues.

As far as tuning of RCA mechanisms is concerned, in order to improve its effectiveness and its pragmatic feasibility within the budget constraints described before, the following actions have been devised:

- Application of RCA as soon as the failure is detected, instead of 'post-mortem' project analysis; this will be accomplished enhancing the defect tracking system used, so that it will be possible to declare and store the 'cause' chosen from a list of pre-defined motivations.

- Extension of RCA also to the failures found during system test, in order to benefit of the feedback before the release is shipped to First Office Application.

- Extension of RCA to scheduling slippages (the activity is currently in progress). 


\section{ACKNOWLEDGEMENTS}

Our thanks go to S. Dal Monte and U. Ferrari who supported and sponsored the initiative. The improvement activities described in this paper were conducted with the support of the Software Quality Rescarch Team and of external consultants. Special mention has to be devoted to: M.C. Aletti, F Aprigliano, M.G. Corti, L. De Domenico, M.L. Giovanelli, M; Giunchi, M.T. Graziani, F. Marziliano, G. Panzeri, F. Pilla, G.F. Pisano, F. Pompili, G. Ru, D. Scrignaro, T. Tucci, G. Vailati.

\section{REFERENCES}

(Arthur, 1992) L.J. Arthur, Improving Software Quality - An insider's guide to TQM. Wiley $($ Internet $=\mathrm{http} / / \mathrm{www}$.wiley.com/ProductInfo.html)

(ASQC, 1995) American Societ for Quality Control, 1995 Malcolm Baldrige Award Criteria, (Internet $=$ http://www.asqc.org)

(AT\&T, 1989) AT\&T, Process Quality Managemet and Improvement Guidelines. AT\&T Quality Steering Committee, Issue 1.1 (Internet = http://www.att.con/infocenter/infores.html)

(Briand, 1995) L. Briand, K. El Emam, W.L. Melo, An inductive Method for Process Improvement: Concrete Steps and Guidelines. Proceedings of ISCN Conference, Wien

(Collofello, 1993) J. Collofello, B. Gosalia, An application of casual analysis to the software production process". In Software Practice and Experience, 23 (10)

(Damele, 1993) G. Damcle, G. Bazzana, M. Giunchi, G. Rumi, Setting-up and using a metrics program for Process Improvement. Proceedings of AQUIS Conference, Venice

(Damcle, 1995) G. Damele, G. Bazzana, M. Giunchi, G. Caielli, M. Maiocchi, F. Andreis, Quantifying the Benefits of Software Process Improvement in Italtel Linea UT Exchange. Proccedings of XV International Switching Symposium

(Furuyama, 1993) T. Furuyama, Y. Arai, K. Iio, Fault Generation Model and Mental Stress Effect Analysis". Proceedings of AQUIS Conference, Venice

(IEEE, 1993) Members of the BOOTSTRAP project team (Editor: D. Card), BOOTSTRAP: Europe's Assessment Method. IEEE Software, May (Internet = http://www.etnoteam.it/bootstrap/index.html)

(Ishikawa, 1991) K. Ishikawa Guida al controllo di qualità. (A guide to quality control) $F$. Angeli

(ISO, 1990) International Organization for Standardization, Quality Management and Quality Assurance Standards - Part 3: Guidelines for the Application of ISO 9001 to the Development, Supply and Maintenance of Software. ISO/IS 9000-3 (Internet = http://www.iso.ch)

(Italtel, 1993) Italtel-SIT-BUCT-Linea UT, Iter di progetto software - Manuale di qualità del processo di produzione di una release software per le centrali della linea UT. Version 04

(Italtel, 1995) Internet www Server location $=$ http://www.italtel.it

(Kuvaya, 1994) P. Kuvaia, J. Simila, L. Krzanik, A. Bicego, S. Saukkonen, G. Koch. Software process assessment \& improvement. Blackwell

(Maggi, 1995) A. Maggi, Il percorso della qualità Italtel (Italtel path to quality).Qualità, $N^{\circ} 1 / 95$

(Nakajo, 1991) T. Nakajo, H. Kume, A case history analysis of software error cause-effect relationship. In IEEE Transactions on Software Engineering, 17 (8)

(Paulk, 1993) M.C. Paulk, CMM for Software - Version 1.1. CMU/SEI-93-TR-24, SEI, (Internet $=$ http://www.sei.cmu.edi)

(Souter, 1992) J.B. Souter, D.P. Cheney, Information Technology Quality System Certification in Europe. Proceedings of 3rd European Conference on Software Quality, Madrid,

(Tosi, 1994) L. Tosi, Analisi dei costi connessi alla qualita' del software (Analysis of costs related to software quality). Graduation thesis University of Milan 


\section{BIOGRAPHY}

G. Damele is Head of the Linea UT Sw Laboratory of Italtel SIT BUCT. He is also Quality Manager for the development projects that have been experiencing Root Cause Analysis.

G. Bazzana is Partner and Consulting Director of Onion, a private company active in the field of communications, technologies and consulting.

F. Andreis is Head of Sw Quality Research for all the business units of Italtel SIT.

F. Aquilio is Manager of the DataBase Sw development area of the Linea UT Sw Laboratory; she is responsible for process improvement activities concerning root cause analysis.

S. Arnoldi is a Senior Researcher at Italtel SIT; he has given continuing support to the definition, implementation and analysis of the RCA program.

E. Pessi is a Senior Consultant at Etnoteam $\mathrm{SpA}$, a major private company that has been supporting the overall process improvement program of Italtel SIT BUCT Linea UT. 